\title{
Efectos de conductas proactivas y prosociales en incidentes críticos de escolares limeños
}

\section{Effects of Proactive and Prosocial Behaviors on Critical Incidents of Schoolers from Lima}

\author{
Jhon A. Holguin Alvarez \\ Universidad Cesar Vallejo, Lima, Perú.
}

\author{
Recibido 07-04-17 \\ Aprobado 01-08-17 \\ En Línea 10-08-17
}
*Correspondencia
Email: jhonholguinalvarez@gmail.com

\section{Citar como:}
Holguin Alvarez, J. (2017). Efectos de conductas proactivas y prosociales en incidentes críticos de escolares limeños, Perú. Propósitos y Representaciones, 5(2), 185 - 244. doi: http:// dx.doi.org/10.20511/pyr2017.v5n2.172

\footnotetext{
El trabajo de investigación proviene del proyecto de investigación en la línea de investigación -Innovaciones pedagógicas-, sección -Educación e Idiomas- (C.R.: 494-17, 2016), del programa de maestría en psicología educacional de la Escuela de Posgrado de Universidad César Vallejo, filial Lima.
}

(c) Universidad San Ignacio de Loyola, Vicerrectorado de Investigación, 2017. 


\section{Resumen}

El estudio se centra en los planteamientos teóricos de conducta proactiva y prosocial de Covey (1996), Xifra (2009) y Roche-Olivar (2004), con el objetivo de analizar las diferencias significativas en la disminución de incidentes críticos en escolares de escuelas públicas y privadas del distrito de San Juan de Lurigancho, para cuyo efecto se aplicaron dos talleres experimentales con metodología cuasiexperimental en tres grupos de estudiantes de $1^{\circ}$ y $2^{\circ}$ de secundaria (G. Exp. (proactividad) $=17$; G. Exp. (prosocialidad) $=$ 15; G. Control=16); se utilizó una bitácora de observación de conducta y el instrumento PANIC de Monereo y Monte (2011). Los resultados indican diferencias significativas con mejores efectos en el taller de conducta proactiva $(\mathrm{Hrp}=16.59, \mathrm{p}<.05)$; a comparación de la dimensión violencia verbal en la cual se obtuvieron mejores efectos por el taller de conducta prosocial $(\mathrm{Hrp}=14.12, \mathrm{p}<.05)$; finalmente, las limitaciones fueron que los estudiantes del taller de proactividad aminoraron sus incidentes críticos demostrando excesivo personalismo, y para posteriores estudios, se sugiere trabajar los talleres mencionados incluyendo a estudiantes agredidos.

Palabras claves: Proactividad, prosocial, incidentes críticos, conducta.

\section{Summary}

The study focuses on the theoretical approaches of proactive and prosocial behavior of Covey (1996), Xifra (2009) and Roche-Olivar (2004), with the objective of analyzing the significant differences in the reduction of critical incidents in students from public and private schools in the district of San Juan de Lurigancho. To this end, two experimental workshops with quasi-experimental methodology were applied to three groups of students of first and second year of secondary education $\left(\right.$ Exp. $\mathrm{G}\left({ }_{\text {proactivity }}\right)=17$; Exp. $\mathrm{G}\left({ }_{\text {prosociality }}\right)=15$; Control G. $\left.=16\right)$; A behavior observation $\log$ and the Guidelines for the Analysis of Critical Incidents (PANIC) instrument of Monereo and Monte (2011) were used. The outcomes indicate significant 
differences with better effects in the proactive behavior workshop $(\mathrm{Hrp}=$ $16.59, \mathrm{p}<.05)$ compared to the verbal violence dimension in which better effects were obtained for the prosocial behavior workshop (Hrp $=14.12, p$ $<.05$ ); Finally, the limitations were that students in the proactivity workshop reduced their critical incidents by demonstrating excessive individualistic. And for later studies, it is suggested to work the above-mentioned workshops, including students attacked.

Keywords: Proactivity, prosocial, critical incidents, behavior. 


\section{Introducción}

Las conductas proactivas y prosociales conducen al ser humano a conseguir propósitos los cuales aportan en la perfección del proyecto de vida desde la etapa escolar en el cambio actitudinal, cognitivo y emocional; y, por ende, mejoran la modalidad de vida de los seres humanos en la sociedad luego de egresar de la escuela. La conducta proactiva es la variable mediante la cual el individuo alcanza distintos niveles de éxito académico y determina la formación del carácter (autoevaluación, decisión y reflexión), por tanto, los factores externos no influyen en su estructuración cognitiva y actitudinal (Covey, 1996; Covey, 2007), es más, en la formación del pensamiento sobre la toma de decisiones, el educando con tal formación, genera su autonomía para alcanzar capacidades y competencias profesionales perdurables en el tiempo.

La conducta proactiva es la expresión y desarrollo de la autoevaluación personal, el análisis de opciones, la toma de decisiones, y la materialización de un lenguaje proactivo. En principio, se entiende que la proactividad establece un marco axiológico que acompaña cada eventualidad que surge en la vida personal o profesional. En educación, es la capacidad para evaluar una situación específica, plantear un propósito con respecto a ella, y mantener la decisión durante un tiempo determinado (figura 1). Dicha decisión se concretiza de acuerdo a la maduración mental de la persona (Covey, 1996), aunque Xifra (2009), describió que estas acciones que organizan las metas y el lenguaje proactivo, estos activan cuestionamientos: qué, cómo; y por qué se realizan las cosas. Aunque hoy en día en el contexto peruano es difícil su inserción pedagógica, se ha dado un inicio en el trabajo basado en la metacognición, pero con gran equivocación, se suele utilizar como un medio de absorción de conocimientos, pero no se instruye para la reflexión, entonces, de acuerdo a los pensamientos relacionados a esta perspectiva, decidir por estimular a los jóvenes en el trabajo proactivo también incluye estimular en el fortalecimiento de las actitudes hacia la vida cómoda, vida 
feliz; o en otros términos: como vida exitosa (Covey, 1996; Torres, Díaz \& Pérez, 2012; Vaello \&y Vaello, 2010).

En el ámbito pedagógico, generar conductas proactivas permitirá que la enseñanza fortalezca a su vez las motivaciones para que los escolares se propongan un modo de vida académica sostenible. Este modelo de enseñanza se manifiesta desde propuestas originarias de la psicología y educación positiva; ceñidas en la formación valorativa de procesos escolares, lo cual recae en el área de tutorización para el logro de mejores interrelaciones escolares y la búsqueda de la satisfacción personal y de otros.

La conducta prosocial es la interacción social que integra el uso de las capacidades humanas para una finalidad colectiva, de apoyo social (Hobfoll \& Stokes, como se citó en Estrella, 1996). En la interiorización y desarrollo de nuevas formas de interrelación, el ser humano materializa: "acciones que benefician a otras personas, grupos... o metas sociales objetivamente positivas" (Roche-Olivar, 2004, p. 39). Como consecuencia, esto promueve la búsqueda de acciones de un conjunto o grupo humano, que, sin interés en la reciprocidad, cooperan para lograr los objetivos colectivos, y cuyas acciones transferidas entre los integrantes de un equipo, motivan hacia la búsqueda de metas que inician desde la escucha activa iniciativa, y cierran el ciclo cooperativo en la escucha activa como reflexión metacognitiva (figura 1). Si se adopta esta definición (González-Portal, 2000); cabe diferenciar que su definición se distingue del proceso altruista ya que las acciones de cooperación no se determinan por recursos inmateriales o motivaciones verbales como ocurre en la prosocialidad. En la escuela, los estudiantes que desarrollan esta conducta, actúan cooperando y a la vez reciben esa cooperación; con relaciones más duraderas y fortalecidas en el espíritu de apoyo socio formativo, es decir, en las acciones realizadas en el aula. 


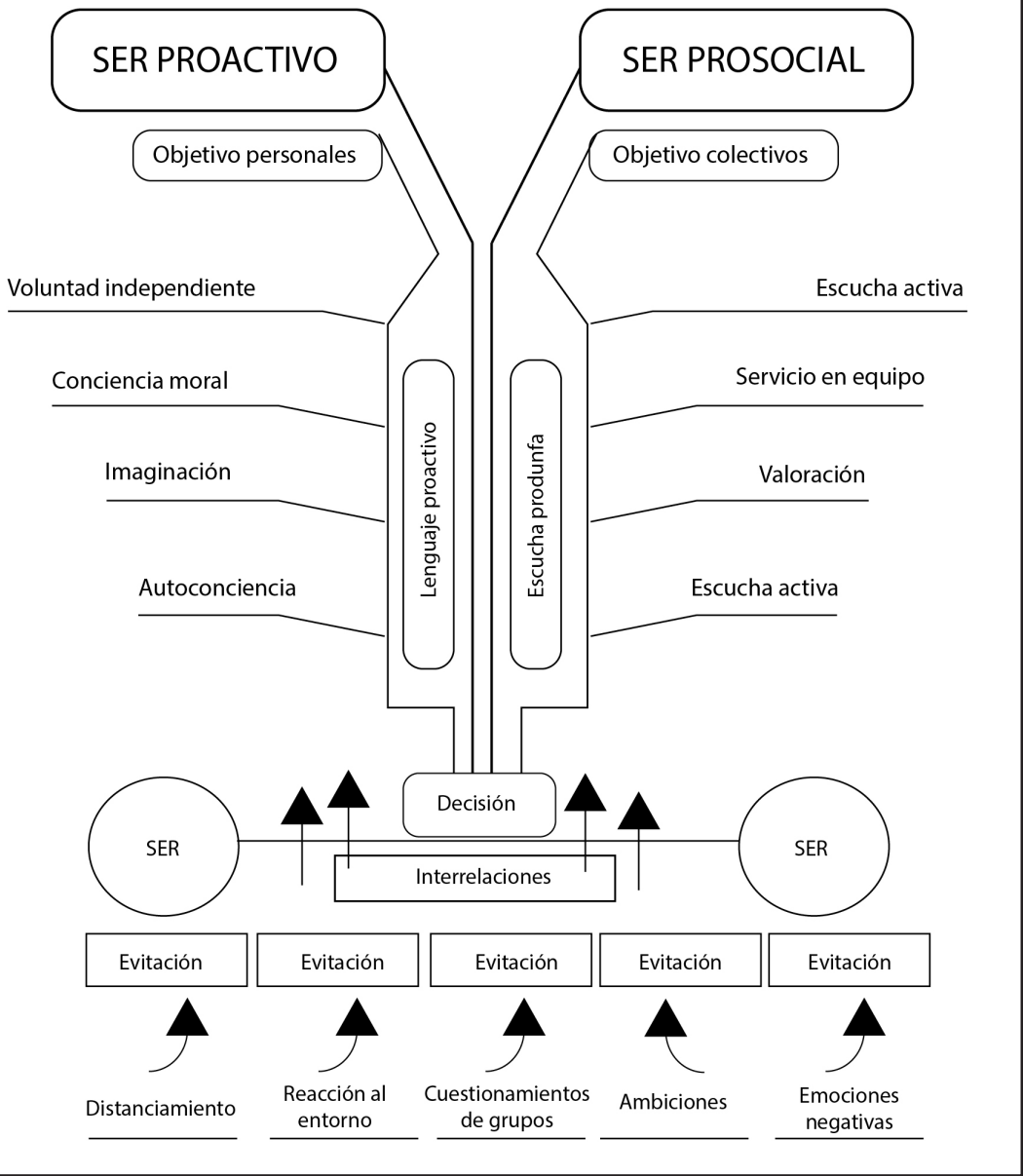

Figura 1. El ser humano necesita de una decisión inicial para el cambio y lograr la evitación de influencias externas. Ante esta decisión el ser podrá madurar acorde a sus objetivos, sean estos personales o colectivos. Gráfica elaborada en base a propuestas de dimensiones de proactividad en "la proactividad definida" de S. Covey (1996, p. 73), y caracteres ligados a la prosocialidad de R. Roche-Olivar (2004, p. 42).

Los incidentes críticos que se caracterizan por la gravedad de ocurrencia, nivel de intensidad, y de latencia; y estos aparecen en contextos determinados; implican la utilidad de agresividad, medios disruptivos en un ambiente de aprendizaje; o son enfrentamientos; desacato de normas estudiantiles por parte del emisor de una conducta. Tripp (2012), las definió como un evento de agresividad en la que se sobrepasan los límites normativos del 
aula y tolerantes de los receptores. Desde esta generación de actitudes en los receptores se puede colegir que el incidente critico es funcional en cuanto aparezca en el proceso educacional un emisor de la conducta y la pasividad del receptor ante el acto de violencia o disrupción, por tanto, es la conducta manifestada a través de la violencia física, verbal y disrupción realizada entre un emisor y receptor de dicha conducta que genera desestabilización cognitiva y emocional en cualquier proceso de interrelación escolar.

Monereo y Monte (2011), describieron al incidente critico como un suceso generador de inestabilidad, originador de la ruptura en la convivencia y en las interrelaciones humanas en el aula, sin embargo, Valdés y Monereo (2012), consideraron que en los receptores de dicha conducta se genera la desestabilización de emocional de la persona en eventos repetidos luego de realizarse por primera vez. Estas conductas pueden cambiar hacia una rebeldía manifestada de forma inusitada, y que generalmente no presenta rasgos con los cuales predecir su aparición. De igual modo, se define como evento de desesperación e inconsistencia estratégica para encontrar soluciones por parte de los tutores o docentes de aula (Every \& Mitchell, como se citó en Bilbao \& Monereo, 2011; Valongo \& Suely, 2006), aunque las últimas investigaciones relatan que desarrollan la reflexión en la práctica docente y se convierte en un medio de aprendizaje en retroalimentación (Monereo \& Monte, 2011; Vallejos, 2013; Valongo \& Suely, 2006).

El origen empírico del incidente crítico, proviene del análisis de un suceso histórico social de John Flanagan en la segunda guerra mundial (Bilbao \& Monereo, 2001; López-Mena, 2011), el cual parte de la examinación de reacciones en pilotos de avión de la segunda guerra mundial que corrían peligro de muerte ante una tragedia latente en el momento Mayday, y de cuyos hallazgos estableció un inicio de la definición psicológica de estos incidentes a los que denominó Critical Incident. Aplicado al campo teórico educacional, el incidente crítico incluye una comunicación activa de tipo agresiva o distractora entre dos o más estudiantes que en el proceso escolar tiene efectos desestabilizadores en el área emocional como cognitiva. 


\section{Problemática y evidencias científicas.}

Las acciones agresivas como la intimidación y el hostigamiento, obstaculizan la convivencia escolar, así como también la disrupción; y esto puede verse desde hallazgos que datan desde los años 2007 y 2008, pues el quinto del tercio superior de la población escolar de China y dos tercios de Zambia generan intimidación escolar en sus aulas educativas (Ministerio de Educación del Perú-MINEDU, 2014, p. 8). En una modalidad similar, el 61\% de estudiantes de América Latina (en Argentina) ridiculizan a sus compañeros, y casi el 48.2\% maltrata a otros estudiantes; y en Brasil, 5 de cada 10 estudiantes se enfrentó a sus profesores de sus escuelas (Eljach, Organización Regional para América Latina y el Caribe-UNICEF, 2011, p. 54-55).

Relacionado con lo descrito, el $45 \%$ de estudiantes de tercer y sexto grado en el Perú fueron violentos con sus profesores, compañeros y materiales de clase; como también fueron disruptivos en el desarrollo de sus clases de aprendizaje (Román \& Murillo, 2011, p. 44); y el 70\% de estudiantes en el año 2014 demostraron agresividad hacia sus docentes en su forma verbal, física y psicológica (El Comercio, 2014, 3er párr.), y desde un análisis determinado por la plataforma Siseve del Ministerio de Educación (Publimetro.pe, 2015), desde el 2014 al 2015, los casos de violencia escolar han aumentado de 1000 a 3000 en las escuelas de distinta gestión. A la actualidad, los reportes de estos casos de incidentes críticos se observaron en tres escuelas del distrito de San Juan de Lurigancho, lo cual fue de interés al ubicar estudiantes de 11 a 13 años de edad que demostraban actos de violencia física, verbal, y de disrupción, lo cual era muy repetitivo; y ante tal realidad, se decidió planificar y elaborar estrategias basadas en la proactividad como en la prosocialidad para aminorar los incidentes críticos en el aula partiendo desde los siguientes cuestionamientos: ¿Los estudiantes que participan en incidentes críticos con buena actitud al cambio, lograrían inhibir esa conducta por el desarrollo de otras de tipo proactiva y prosocial? ¿Qué efectos desarrollaría un taller de tutorización aplicado durante un tiempo determinado en un grupo de jóvenes agresores y disruptivos? 
Para responder a estas cuestiones se revisaron las evidencias empíricas como: Herfst, van Oudenhoven y Timmerman (2008), reportan que el rechazo e intolerancia a las distintas culturas puede identificarse de forma clara utilizando la técnica de evaluación de incidentes críticos, y remiten que la valoración reflexiva de las culturas puede realizarse en el alumnado desde el desarrollo de un pensamiento intercultural. En cambio, el trabajo de Vandercleyen (2010), demostró que los estudiantes franceses los cuales desarrollaron estrategias de control emocional, cuando participan de actividades mediadas por una buena comunicación, logran integrarse a actividades conjuntas para el aprendizaje del desarrollo corporal y de convivencia en sus escuelas.

Shuster (2009), en su estudio social analizó los cambios en los grados de violencia en Estados Unidos, encontrando 37 incidentes en base a utilización armas como parte de un delito escolar, por lo cual reporta a un 93\% del total de sujetos que portaban armas en el estado de Colorado con signos de desesperación y estrés. Sin embargo, las evidencias cualitativas sobre incidentes críticos también aportan a la realización de la investigación internacional como el estudio realizado en Concepción, Chile, de Muñoz y Nail (2013), en el cual declaran a 15 profesores, quienes encontraron que los factores que son más predictores de la aparición de los incidentes críticos se hallaban centrados en la falta de claridad en normativas escolares, su poca falta de inculcación en ellos durante las actividades escolares; o en determinado momento llegaban a ser restrictivas y excluyentes de grupos que adolecían de distintas conductas de enfrentamiento; y esto parece haberse relacionado con el estudio de Nail, Muñoz y Gajardo (2013), quienes declaran que el procedimiento más usual para desarrollar incidentes críticos se efectúan desde el acercamiento, tratamiento del receptor de la conducta, y desequilibrio de las normas; por lo que existe mayor inflexibilidad en el control emocional de los emisores de dicho comportamiento.

Las experiencias de Ison-Zintilini y Morelato-Giménez (2008), al igual que la de Valdivieso (2009), refieren que la violencia escolar está 
relacionada con el grado de amistad existente entre los el grupo de agresores potenciales que incluyen a los agresores ya iniciados en el proceso escolar; y a su vez, el estudio de Desbiens et al. (2008), revela que tras aparecer los incidentes críticos en el proceso escolar, es más rápida la aparición de alerta hacia el peligro en los más desfavorecidos; y en algunos alumnos que son pertenecientes a ese grupo, estos generan modalidades de defensión o rechazo social hacia los agresores, lo cual genera caos en las relaciones del alumnado; y si estas conductas se hacen repetitivas, los docentes pierden el control fácilmente y evitan resolver el problema surgido, lo cual afecta a la sana interacción de grupos.

En Callao (Lima), Gordillo (2013) y Gordillo y Gamero (2013), describen resultados que establecen que la disrupción está más presente en escuelas de género mixto, y se multiplica en el alumnado por imitación, pero existe cierta moderación cuando en los grupos generadores de incidentes críticos se incluyen estudiantes del sexo femenino.

Respecto a proactividad Gustems y Calderón (2014), manifiestan que por conductas proactivas los estudiantes desarrollan mejores emociones en situaciones críticas cuando practican la reflexión directa de los hechos, por lo que el evitamiento desaparece gradualmente, y en cierta medida, esto concuerda con lo expuesto por Sanz, de Miguel y Gómez (2014), quienes remiten que el deseo de control desarrolla autonomía en los estudiantes que agreden, por lo cual se forman como personas más decisivas después del cambio al desarrollarse de forma autónoma, debido a que superan el miedo ante una nueva ocurrencia critica como un incidente de violencia o disrupción.

Por último, Cuevas (2012) trabajó con estudiantes de 14 a 17 años de edad del distrito de San Juan de Lurigancho, en cuyo estudio encontró una relación significativa entre la conducta prosocial y el clima social familiar, lo cual indicó que las relaciones, desarrollo y estabilidad de estudiantes dependían de puntajes positivos en la variable clima social en familia, también evidenció la conducta prosocial en la interacción familiar de los estudiantes, 
quienes demostraron alto nivel de desarrollo de valores de acuerdo a su estilo de convivencia familiar.

\section{Hipótesis.}

La hipótesis que intentó responder los cuestionamientos iniciales, parte del planteamiento de proactividad de Covey (1996), basado en el desarrollo de competencias y actitudes de formación humana proactiva como también en el planteamiento de Roche-Olivar (2004) sobre inteligencia prosocial y conductas prosociales, las cuales se desarrollarían en los estudiantes de 11 a 12 años de edad vinculados a incidentes críticos durante más de un año escolar, buscándose inhibir la conducta incidente crítico por un tratamiento experimental, para desarrollar:

-Competencias proactivas (1er grupo):

Aptitudes de autoconciencia, imaginación, conciencia moral y voluntad independiente.

-Competencias prosociales (2do grupo):

Aptitudes de apoyo mutuo, escucha activa, valorización y servicio en equipo.

El tratamiento transferido por actividades pedagógicas a los estudiantes, tales como la participación por equipos, trabajo individualizado, desarrollo de estrategias conversacionales y de autocontrol emocional; comprobaría que dichas actividades desarrollaron el control de emociones manifestadas en la ocurrencia de sus incidentes, y cuyos efectos manifestarían mejora en cada grupo de escolares, como también saber cuál de ambos tratamientos fueron más efectivo, luego de contrastar los resultados con estudios con similar enfoque (Desbiens et al., 2008; Vandercleyen, 2010). De lo cual se desprendió la hipótesis:

hl $=$ Existen diferencias estadísticamente significativas en los incidentes críticos de los estudiantes que asistieron a los talleres de conducta proactiva y prosocial de las Instituciones Educativas de San Juan de Lurigancho. 


\section{Método}

El método es hipotético deductivo, ya que busca contrastar una hipótesis desde deducciones por inferencias estadísticas (Sabariego \& Bisquerra, 2004). En este caso se plantearon hipótesis sobre los efectos en los incidentes críticos, que se asumieron disminuirían luego del tratamiento, a través de talleres experimentales de conducta proactiva y prosocial.

La investigación es aplicada (Babbie, 2000), ya que se aplicaron actividades pedagógicas para establecer conductas determinadas que generen cambios en dos grupos de escolares de un contexto determinado, que en este caso fueron sujetos de 11 a 13 años de edad del distrito de San Juan de Lurigancho en Lima. En cuanto al diseño se utilizó el tipo cuasi experimental con medición pre y pos prueba (Balluerka \& Vergara, 2002); en cuyo procedimiento se realizó la manipulación de dos variables independientes (Cook, como se citó en Balluerka \& Vergara, 2002): conductas proactivas y conductas prosociales.

\section{Participantes.}

La muestra se compuso por 48 estudiantes de 11 a 13 años de edad $(\chi=+-12$; $\mathrm{E}_{(\text {máx.) }}=13 ; \mathrm{E}_{(\text {min.) }}=11$ ), organizados en tres grupos correspondientes a tres escuelas públicas del distrito San Juan de Lurigancho de la capital de Lima. Estos se agruparon en función a la orientación pedagógica de cada taller: grupo $\mathrm{A}(\mathrm{t}$ proactivo $)=17$ estudiantes, grupo $\mathrm{B}(\mathrm{t}$ prosocial $)=15$ estudiantes; y un grupo control= 16 estudiantes. La selección se realizó de acuerdo al criterio de participación de los estudiantes en más de tres ocasiones en incidentes críticos en más de dos meses iniciales del año escolar lo cuales fueron reportados por los docentes al departamento psicológico de cada escuela. Otros criterios de inclusión en el experimento fueron:

- Decisión del estudiante por realizar un cambio en su conducta.

- Pertenecer a la tutela o estar contacto con un docente desde un año anterior al estudio. 
- Paternidad y profesorado: aceptar a los alumnos cuyos padres y profesores mostraran disposición para que el estudiante participe de las actividades.

En cada grupo de escolares, las cantidades por género también presentaron cantidades homogéneas para el experimento $\left(\mathrm{G}_{(\text {femenino) }}=53 \%\right.$ y $\mathrm{G}_{\text {(masculino) }}=$ $47 \%$ ). Cabe señalar que las muestras incluían individuos repitentes en por lo menos un grado de escolaridad; sin embargo, las investigaciones en la que se incluyen estudiantes con estas características no reportan resultados que sugieran invalidez externa o interna (Muñoz \& Nail, 2013; Vandercleyen, 2010; Vallés \& Monereo, 2013). En esta investigación se incluyó como unidad informante a 15 profesores que aportaron en el recojo de datos (cinco por cada escuela); con el fin de que dichos datos se obtuvieran de forma veraz y confiable mediante la cercanía a sus propios alumnos y el manejo tutorial en cada grado.

\section{Instrumentos y procedimiento.}

Reporte de incidentes críticos (REPIC): Escala cuantitativa, elaborada para su aplicación en la investigación, estructurada por 20 ítems orientados a medir tres tipologías de incidentes críticos determinados por su aparición en el aula: violencia física, violencia verbal, y disrupción. La frecuencia de su aparición se estableció a través de una escala de respuestas por cada oportunidad de aparición del incidente en el aula, patrio de recreo, o en otra instalación de la escuela: Siempre, casi siempre; y a veces. Los puntajes asignados sumaban un total máximo de 60 y mínimo de 20.

La validación del instrumento se desarrolló por juicio de dos expertos en violencia y bullying escolar; y cuatro profesores especialistas en el área de psicología educacional. El proceso constó de correcciones y ajustes escalonados a posteriori. Es decir, dos jueces evaluaron una primera versión del instrumento sobre la redacción de ítems y su correspondencia a cada dimensión, luego de este proceso, dos profesores especialistas en la temática evaluaron su corrección los cuales dieron pase a otros dos profesores 
especialistas ( $\sin$ conocer identificación), para la evaluación de la versión final.

El proceso facilitó indagar si los procesos primarios de validación eran rigurosos en cuanto a opinión especializada por el juicio de expertos y obtener resultados más coherentes en la evaluación. Luego de este proceso, se calculó el índice de validez de contenido obteniéndose $98 \%$ de aceptación inicial y 95\% de aceptación final, lo cual se aceptó para desarrollar la aplicación del instrumento.

La fiabilidad se calculó desde los datos obtenidos tras la realización de una prueba piloto con 45 sujetos con idénticos problemas de incidencia critica, por lo que se extrajeron puntuaciones para el cálculo del índice Alfa de Cronbach en: Violencia física $=.788$; violencia verbal $=.769$; disrupción $=$ .781 ; y la variable incidente críticos $=.759$. Esto permitió utilizar el instrumento con total fiabilidad.

Pauta de incidentes críticos (PANIC): instrumento elaborado por Monereo y Monte (2011), dirigido para uso del docente tutor y profesor de polidocencia, con el fin de evidenciar las conductas manifestadas por los estudiantes del experimento, y que sea un apoyo para reportar las conductas con total claridad en un evento determinado; y también como método de registro de conductas muy específicas; por tal, el instrumento permitió recoger datos como: a) descripción del contexto, b) descripción del IC, c) concepción del docente y del alumno, d) sentimientos asociados; y e) acciones tomadas y resultados. Este instrumento se introdujo como parte de la bitácora de los docentes de cada escuela.

Talleres t proactivo y t prosocial: Estructurados por 25 actividades por cada taller (sesiones de tutorización), según las competencias de cada programa curricular, y se desarrollaron durante seis meses de actividad curricular. Cada sesión se realizó de 25 a 35 minutos durante las horas lectivas del área de personal social y tutoría. En el taller t proactivo se desarrollaron actividades para estimular aptitudes de acuerdo a cada dimensión de cada variable (tabla 
1). De igual modo, se desarrollaron actividades y técnicas por cada taller acercándose al desarrollo de las dimensiones de valor independiente en la investigación.

Como primer paso de la investigación, se realizaron acuerdos con las autoridades de las escuelas implicadas en el estudio. Se establecieron actividades por cada área de tutoría y se propuso a cada docente que sus estudiantes desarrollasen sus clases sin que se señalase que iban a ser evaluados por observación. En un segundo momento, se realizó un chek list para organizar con los docentes a los estudiantes vinculados a más de tres oportunidades a incidentes críticos de tal manera que este procedimiento facilitase la agrupación de alumnos (t proactivo y t prosocial). A partir de este listado también se organizó la aplicación piloto con los docentes de las Instituciones Educativas. 


\section{Tabla 1.}

Actividades experimentales aplicados por cada taller $T$ proactivo y $T$ prosocial para niños y niñas de 11 a 13 años de edad relacionados a incidentes críticos.

\begin{tabular}{|c|c|c|c|}
\hline $\begin{array}{c}\text { Taller de } \\
\text { proactividad }\end{array}$ & Actividades y técnicas & $\begin{array}{c}\text { Taller de } \\
\text { prosocialidad }\end{array}$ & Actividades y técnicas \\
\hline 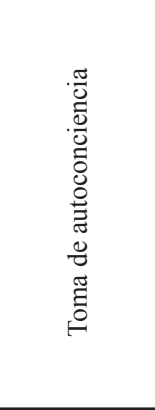 & $\begin{array}{l}\text { FODA personal: } \\
\text { Autoevaluación de los actos: } \\
\text { Evaluación del entorno } \\
\text { Análisis FODA personal } \\
\text { Autoconocimiento de faltas } \\
\text { Reincorporación de los valores: } \\
\text { Organización de acciones } \\
\text { autorreguladoras: auto } \\
\text { cuestionamiento } \\
\text { Fortalecimiento del espíritu de } \\
\text { cambio }\end{array}$ & 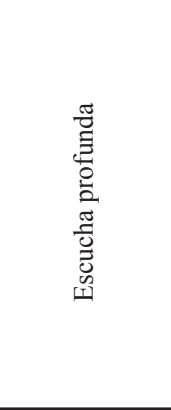 & $\begin{array}{l}\text { Dinámicas de integración de } \\
\text { grupos } \\
\text { Análisis y reflexión grupal de } \\
\text { acciones críticas realizadas }\end{array}$ \\
\hline 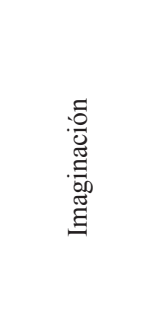 & $\begin{array}{l}\text { Creación de oportunidades: } \\
\text { Programación de actividades } \\
\text { semanales de auto superación } \\
\text { Asignación conversacional del } \\
\text { acompañante } \\
\text { Aplicación de V de Gowin para } \\
\text { creencias, realidades, acciones y } \\
\text { compromisos }\end{array}$ & 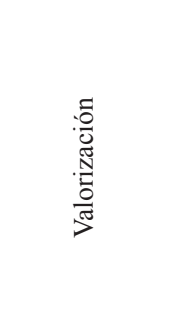 & $\begin{array}{l}\text { Publicidad escolar: Creación } \\
\text { de posters y afiches alusivos al } \\
\text { trabajo compartido } \\
\text { Evaluación de acciones } \\
\text { compartidas en sociedad } \\
\text { Reflexión estudiantil en la } \\
\text { web de grupos por Institución } \\
\text { Educativa }\end{array}$ \\
\hline
\end{tabular}

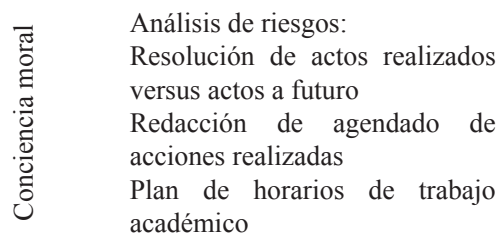

Aplicación de técnicas de
liderazgo por equipos
Apoyo social de servicios de
salud por seminarios y foros
estudiantiles

$\begin{array}{ll} & \begin{array}{l}\text { Recojo de opiniones: } \\ \text { Análisis del estado comunicativo }\end{array} \\ & \text { Dirección de actividades: Foro } \\ \text { Relatos personales de } & \text { experiencias eficaces de trabajo } \\ \text { valorativo o académico }\end{array}$

En un tercer paso, se organizó la evaluación pretest y postest de los estudiantes, en una entrevista personal con cada docente informante de aula 
(tutor o polidocente). Y con el fin de establecer medios que no influyeran de forma externa a la investigación cada docente trabajó con dos bitácoras de anotaciones de conductas. Una de ellas serviría para registrar los puntajes y características que los estudiantes alcanzaban en la realización del trabajo en grupo tal cual establecía el Ministerio de Educación del Perú en las carpetas pedagógicas del docente; y otra bitácora, serviría de registro de las características esenciales y puntajes a los instrumentos consensuados con el equipo psicológico de las escuelas. Los estudiantes tampoco tuvieron conocimiento del registro auxiliar con los instrumentos mencionados.

En el cuarto paso, los docentes de aula acordaron con los estudiantes que mostraban decisión de cambio en los últimos meses, por cuanto se les invitó a participar de sesiones de tutoría con un docente invitado de otra institución educativa con el fin de trabajar ideas compartidas del trabajo por cada taller. Las sesiones se trabajaron con total normalidad en cuanto al tiempo de trabajo en los dos grupos de experimentación. Cabe señalar que de dos a una semana antes de culminar los talleres, los docentes a cargo registraron en tres o cuatro oportunidades los datos por instrumento; tratando de captar los puntajes y características más cercanas a la realidad. Finalmente, se calcularon las diferencias de datos, utilizando pruebas estadísticas bajo criterio de normalidad de datos; y para corroborar dichas diferencias se utilizaron pruebas estadísticas paralelas.

\section{Resultados}

La comprobación estadística se realizó a través de la prueba Kruskall Wallis, desde el contraste de diferencias entre las puntuaciones pre y postest, de lo cual se obtuvo 8 puntos de diferencia en el rango promedio de la variable incidentes críticos en el grupo t proactivo, y de 0.53 en el grupo t prosocial. Por otro lado, se evidenció en la misma variable un aumento de 9 puntos en el aula grupo control, corroborándose con un $95 \%$ de nivel de confianza (pretest (sig.) $_{\text {(n) }}$ $=.470$ postest $_{\text {(sig.) }}=.000, \mathrm{p}<.05$ ). Para comprobar estas diferencias con una prueba paramétrica también se utilizó un Anova con índices significativos 
$\left(\mathrm{F}_{\text {(pretest) }}=.089 ; \mathrm{F}_{\text {(postest) }}=19.862, \mathrm{p}<.05\right)$. Es decir, no existieron diferencias antes de aplicar los talleres, lo que demostró la estabilidad estadística entre grupos antes de iniciar la aplicación de los talleres respectivos.

Ante estos hallazgos iniciales, se realizó una prueba post hoc Dunnett para definir la efectividad entre talleres, comprobándose su significancia estadística. Al respecto, se obtuvo que el taller t proactivo fue más efectivo que el taller t prosocial en la variable incidentes críticos $\left(\mathrm{m}_{(\mathrm{t} \text { proactivo })}: 37.06\right.$, $\mathrm{p}<.05$ ); $\mathrm{y}$ en último lugar, quedó el grupo control. En la dimensión violencia verbal el taller t prosocial fue más efectivo que el taller proactivo $\left(\mathrm{m}_{(\mathrm{t} \text { prosocial) }}\right.$ : $12.6, \mathrm{p}<.05$ ), y en las dimensiones restantes (violencia física y disrupción) las comparaciones presentaron mayores efectos en alumnos del taller $\mathrm{t}$ proactivo.

Para diferencias de efectividad por taller en cada dimensión, el Anova de un factor arrojó diferencias significativas $\mathrm{F}_{\text {(violencia fisica) }}=18.445, \mathrm{p}<.05$; violencia verbal $\mathrm{F}_{\text {(violencia verbal) }}=24.177, \mathrm{p}<.05 ; \mathrm{F}_{\text {(disrupción) }}=7.059, \mathrm{p}<.05$ ). Habría que notar la inexistencia de diferencias antes de la aplicación de los talleres en dichas dimensiones, lo cual sustenta la estabilidad de equivalencia estadísticas en las puntuaciones. Luego de corroborar diferencias en la medición postest en todas las dimensiones, se aceptó la hipótesis formulada inicialmente sobre la inhibición del incidente critico desde la generación de conductas proactivas y prosociales. 


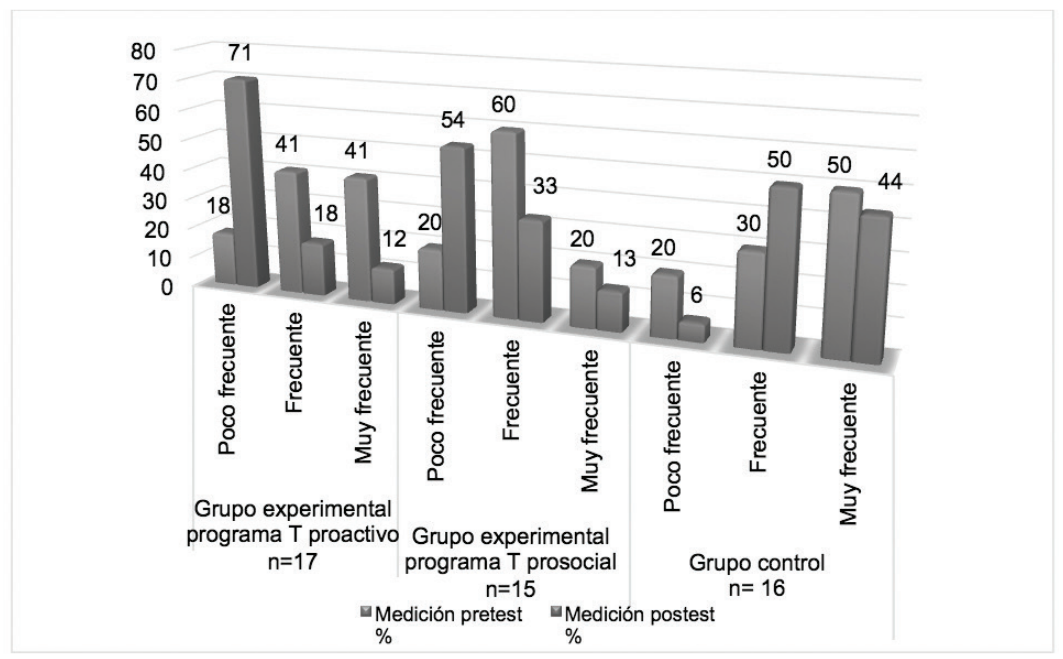

Figura 2. Porcentajes de incidentes críticos en medición pre y postest de los grupos experimental y control de tres escuelas públicas de San Juan de Lurigancho, 2016.

La descripción de frecuencias porcentuales de los incidentes críticos, los sujetos del grupo control presentaron incidentes críticos muy frecuentes en casi el $50 \%$ antes de aplicar los talleres de tutorización $\mathrm{t}$ proactivo y $\mathrm{t}$ prosocial (figura 2). En las mediciones por alumnado, en el grupo t prosocial el $60 \%$ de los participantes presentaron incidentes críticos frecuentes y en el $20 \%$ de forma frecuente. Es decir, que en el grupo t proactivo adicionando las cifras de frecuencia, el $80 \%$ del total de sujetos de ese grupo presentaron por lo menos incidentes críticos frecuentes o repetitivos; lo que determinó inicialmente que los estudiantes de este grupo presentaron violencia de tipo física (golpes, empujones, entre otros), violencia verbal (insultos, gritos injustificados, enfrentamientos); y disrupción (interrupciones de clase en conversaciones con otros, distracciones de compañeros; etcétera).

En la descripción postest, en el grupo control, se presentó una leve disminución de los incidentes en el 6\% del total de sus participantes. En el grupo del programa taller t prosocial la disminución fue mucho mayor con $25 \%$ en el nivel de frecuencia. Sin embargo, se presentó una disminución mucho mayor en el grupo del taller t proactivo, estableciéndose el 29\% de incidentes críticos muy frecuentes. Es decir, la disminución de estos 
incidentes en los estudiantes del grupo t proactivo fue de más del $20 \%$ en aquellos que presentaban esta conducta con frecuencia como también con mucha frecuencia.

\section{Discusión}

El estudio abordó la realidad estudiantil de tres instituciones educativas, de las cuales dos grupos recibieron tratamiento a través de talleres experimentales con el fin de disminuir los incidentes críticos en su interacción de aula. Los datos corroboraron con las pruebas a posteriori, que los asistentes del taller proactivo manifestaron diferencias significativas en los incidentes críticos; lo cual se estableció desde técnicas y actividades aplicadas desde los talleres de tutorización.

Desde la mirada pedagógico tutorial, se aduce que las estrategias cognitivas personalistas del taller T proactivo, contribuyeron en la reflexión de los participantes, en su autoevaluación, planeación de técnicas para la mejora de sus acciones de vida, el uso del lenguaje proactivo, como también la organización de actividades académicas según sus propias limitaciones y fortalezas académicas y, por último, mejorar la toma de decisiones. La hipótesis planteada confirmada parece asentarse en la búsqueda de interacción desarrollada por la comunicación basada en principio, por el buen uso del lenguaje, esto indicó la mejora de la percepción del alumnado de los mensajes entre estudiantes al finalizar de proactividad; y eso se asemeja a lo revelado por el estudio de Vandercleyen (2010), quien descubrió que al lograr un lenguaje proactivo, los sujetos con manifestación de incidencia critica acomodan mejores técnicas para interrogar, organizarse ellos mismos, e integrarse a grupos que naturalmente cuyos integrantes los rechazan, de igual modo, estas evidencias se sustentan los planteamientos teóricos de Xifra (2009), Covey (1996); y Covey (2007); lo cual advierte que los sujetos lograron autoevaluarse y fundamentar utilizar un lenguaje apropiado para comunicarse, sino también para adaptarse a la interacción o ambiente conversacional de los integrantes de este grupo; lo cual también ocurriría si 
el taller se hubiese desarrollado con alumnos afectados (agredidos), lo cuales no participaron en el taller, aunque hay que tener en cuenta una principal limitación: los grupos experimentales solo se conformaron por alumnos agresores o disruptivos y no por agredidos; y al respecto Herfst et al. (2008), plantean que para desarrollar la conducta proactiva se necesita adecuar un ambiente positivo, lograr competencias como la empatía y así fortalecer la iniciativa cultural o deseos de culturización, lo cual ha ocurrido en este grupo de alumnos, en los cuales la maduración también ha jugado un aspecto positivo que se reflejó en la actitud de cambio de los participantes, y luego estadísticamente con mejores índices significativos en la comparación de puntajes obtenidos desde la aplicación del instrumento.

Los estudios referenciados se han centrado en el análisis de incidentes críticos basados en problemas de interculturalidad, es decir, en alumnos con variada tipología de los incidentes críticos: los que prodigan el rechazo hacia el alumnado de acuerdo a un origen étnico, alumnos con condiciones económicas distintas; y aquellos con creencias religiosas no católicas; es necesario acotar con que son experiencias de otros contextos Francia, Alemania, Austria, Holanda, y Bélgica, lo cual en parte ha sido crucial para acercar la realidad al lugar de la investigación realizada; más por las características de las conductas y los rasgos culturales que el factor socioeconómico, sin embargo, otra asunción a realizar es que los sujetos del experimento, presentaron al inicio de las actividades poca tolerancia para la actividad académica, sin desarrollar estrategias de autorregulación académica, lo que explicita Alegre (2014) como un elemento esencial para mantener una motivación adecuada en la actividad académica y así evitar estímulos externos, y extrapolando al estudio, estos estímulos se deben a influencias de otros agresores con mayor poder de convencimiento en la interrelación social de aula, lo cual debilita mantener las decisiones tomadas desde un inicio.

Las evidencias empíricas que sirven de comparación metodológica a los incidentes críticos a nivel internacional, centran su atención a condiciones de 
tipo: violencia social y delincuencia juvenil (Shuster, 2007), o en situaciones de riesgo juvenil en donde el pandillaje es un elemento central (Desbiens et al., 2008; Villacorta, 2014), por tanto, en la realidad de los alumnos del distrito San Juan de Lurigancho, la delincuencia y pandillaje también fueron variables que acompañaron el desarrollo de los estudiantes y por ende han sido fuentes de iniciación en incidentes crítico, pues muchos, antes de comenzar los talleres, presentaban conductas de enfrentamiento y disrupción aunada a la agresividad, aunque esto fue en incremento hasta los meses antes de la dicha aplicación, el problema se debería al apego escolar a los amigos de barrio, quienes viven en situaciones de riesgo, aunque la condición laboral que los niños presentan se visualiza en las calles donde corren peligro, y ello contrae interactuar con jóvenes de la zona que integran grupos de pandillaje o delincuencia, por otro lado, estudios similares al realizado, se estudiaron variables más personales como la agresividad parental, lo cual causa incertidumbre en los docentes por no encontrar una solución al problema como también sucedería en los familiares de los implicados (Bilbao y Monereo, 2011; Contreras, Monereo \& Badia, 2010; Nail, Muñoz \& Gajardo, 2013; Muñoz \& Nail, 2013); si bien es cierto, los docentes de las Instituciones Educativas presentaron rechazo, fastidio y hartazgo sobre los estudiantes también disruptivos, esto se dilucidó en el gran porcentaje de los puntajes obtenidos de la observación de la conducta disruptiva, aunque también el progreso moderado por la efectividad del taller t proactivo también remarcó las diferencias ya analizadas a nivel de variable.

En la violencia verbal la comparación estadística indicó que la inhibición de los incidentes críticos en los escolares provocara en los estudiantes mejor servicio en equipo, valorización de sus compañeros y también la escucha activa, lo que se logró a través del taller T prosocial, y que se logró gracias a la implementación de los planteamientos de Xifra (2009) y Roche-Olivar (2004), lo cual proviene de la conducta proactiva, no obstante, es base teórica del taller t prosocial, en la cual se buscó desarrollar aspectos de ayuda mutua y trabajo colectivo, y estas actividades se aproximaron a los aportes 
experimentales de Garaigordobil y Berrueco (2007), quienes plantearon estrategias de cooperación, juego amistoso, ayuda mutua y uso del buen lenguaje con resultados efectivos, y en el taller T prosocial, aplicado para la presente investigación, los sujetos se evaluaron respecto a sus acciones con sus compañeros y todo partió desde el análisis de los medios que utilizaban para comunicarse, y como efecto, esta maduró en realizarse sin utilizar groserías o gritos injustificados, y desde aspectos de competencia valorativa, lograron proponerse por su propia decisión, actividades como por ejemplo, alcanzar mejores promedios que los de sus compañeros con altas calificaciones que no participaban del taller, agendar una rutina de estudio o plantear un medio de autoevaluación como técnica para manejar la interacción con otros.

Finalmente, es necesario acotar que los tratamientos aplicados para disminuir incidentes críticos en el aula, se diferenciaron por la naturaleza del propósito de actitudes a desarrollar y las herramientas cognitivas de autocontrol, lo cual justifica el desarrollo de conductas proactivas como la autoevaluación, análisis de debilidades y fortalezas, y toma de decisiones, siendo técnicas que requieren un manejo independiente de las actividades académicas al evitar influencias de otros; o lo cual también podría recaer en la imitación de la disrupción, sin embargo, una de las limitaciones encontradas también se centra en el individualismo que provocó el tratamiento del grupo experimental t proactivo al disminuir los incidentes críticos, lo que fue obstáculo para mejorar relaciones socio afectivas; lo que si se manifestó con mayor aplomo en las conductas prosociales desarrolladas en el taller $\mathrm{t}$ prosocial, aunque sus efectos hayan secundado a los del taller de proactividad.

\section{Conclusiones}

Las diferencias estadísticas en los incidentes críticos entre el grupo de participantes del taller $\mathrm{t}$ proactivo, $\mathrm{t}$ prosocial y grupo control, indican mejores efectos en alumnos del taller t proactivo ( $\mathrm{h}$ sig. $=.000, \mathrm{p}<.05$ ).

Se comprobó la disminución de incidentes críticos con mayor efectividad en sujetos que asistieron al taller $\mathrm{t}$ proactivo con mayor promedio ( $\mathrm{m}$ : 
37.06); y las actividades del taller t prosocial fueron efectivas en segundo lugar en esta comparación ( $\mathrm{m}: 39.13, \mathrm{p}<.05$ ), cuyos resultados permiten aceptar la hipótesis inicial, y deducir que el modelo teórico fundamentado en las capacidades proactivas de Covey (1996) fueron más efectivas que la conducta prosocial desarrollada bajo postulados de Roche-Olivar (2004).

La disminución también fue significativa en las dimensiones violencia física y disrupción, siendo en ambas más efectivas las actividades del desarrollo conductual proactivo por actividades tutoriales a diferencia de la conductuación prosocial $\left(\mathrm{m}_{\text {(violencia física) }}=9.882 ; \mathrm{m}_{\text {(disrupción) }}=13.529, \mathrm{p}<.05\right.$ ), esto reveló que ciertas conductas agresivas y disruptivas que disminuyeron por establecerse objetivos personalistas con mayor incisión en los participantes del taller t proactivo.

En la violencia verbal la disminución también fue significativa, presentando mejores efectos en los promedios de los asistentes del taller $\mathrm{t}$ prosocial ( $\mathrm{m}: 12.6, \mathrm{p}<.05$ ), cuyo hallazgo establece que las actividades de tipo prosocial incrementan el ajuste de la personalidad de los estudiantes hacia la participación grupal, en la cual mejoraron sus relaciones interpersonales desde la escucha activa y la valorización.

Por último, el método de incidentes críticos estableció un inicio en el control emocional en los docentes a través del lenguaje proactivo y la escucha activa que los estudiantes presentaban paulatinamente en sus aulas según sus manifestaciones cotidianas con los docentes a cargo de los talleres; y también aceptaron que la verificación de incidentes críticos es una experiencia reflexiva para el docente relacionado a la problemática en investigación.

\section{Referencias}

Alegre, A. (2014). Autoeficacia académica, autorregulación del aprendizaje y rendimiento académico en estudiantes universitarios iniciales. Propósitos y Representaciones 2(1), 79-120. https://doi.org/10.20511/ pyr2014.v2n1.54 
Ares, A. (2004). La conducta de los emprendedores (pp. 493-498). Portularia 4. Recuperado de http://rabida.uhu.es/dspace/handle/10272/179

Babbie, E. (2000). Fundamentos de la investigación social. México D.F.: Thomson Editores.

Balluerka, N., \& Vergara, A.I. (2002). Diseños de investigación experimental en psicología. Madrid: Pearson Educación.

Bas, E. (2014). Educar para innovar: la innovación como cultura. Juventud, proactividad, creatividad, participación y visión de futuro compartida. Revista de Estudios de Juventud, 14(104), 11-30.

Bilbao, G., \& Monereo, C. (2011). Identificación de incidentes críticos en maestros en ejercicio: propuestas para la formación docente. Revista Electrónica de Investigación Educativa 13(1), 131-151. Recuperado de: http://redie.uabc.mx/vol13nol/contenido-bilbaomonereo.html

Comité de seguridad ciudadana-Codisec Sjl (2016). Plan local de seguridad ciudadana 2016. San Juan de Lurigancho. Recuperado de: http://munisjl. gob.pe/1/wp-content/uploads/2016/01/informe-de-avance-plsc-2016final.pdf

Contreras, C; Monereo, C., \& Badia, A. (2010). Explorando la identidad: ¿Cómo enfrentan los docentes universitarios los incidentes críticos que ocurren en las aulas de formación de futuros profesores? Estudios pedagógicos, 23(2), 63-81. https://doi.org/10.4067/S071807052010000200004

Covey, S. (1996). Los 7 hábitos de la gente altamente efectiva. Barcelona: Paidós Ibérica.

Covey, S. (2007). Los 7 hábitos de los adolescentes altamente efectivos. México D.F.: Grijalbo.

Cuevas, L. (2012). Clima social familiar y conducta prosocial en estudiantes de 3ro, 4to y 5to de secundaria del distrito de San Juan de Lurigancho (Tesis de licenciatura). Universidad César Vallejo, Lima.

Desbiens, J.-F., et al. (2008). Une analyse des comportements perturbateurs survenus durant des cours d'éducation physique et à la santé enseignés par des stagiaires. Staps,3(81),73-88. https://doi.org/10.3917/sta.081.0073 
Eljach, S., Organización regional para América Latina y el Caribe-Unicef (2011). Violencia escolar en América Latina y el Caribe: Superficie y fondo. Unicef: Panamá. Recuperado de: http://www.unicef.org/

El Comercio (agosto, 2014). Más de mil casos de violencia escolar en Lima en el último año. Recuperado de: http://elcomercio.pe/lima/sucesos/ mas-mil-casos-violencia-escolar-lima-ultimo-ano-noticia-1752801

Escribano, L., González, A., Ortiz, M., Simón, C., Tarragona, M., \& Uribe, E. (2010). La prevención de conductas desafiantes en la escuela infantil. Un enfoque proactivo. México. Recuperado de: http://www. asociacionalanda.org/pdf/Libro.pdf

Estrella, J. (1996). La teoría del apoyo social y sus implicaciones para el ajuste psicosocial de los enfermos oncológicos. Revista de Psicología Social, 6(2), 257-271. Recuperado de: https://dialnet.unirioja.es/ ejemplar/10818

Garaigordobil, M. (2014). Conducta prosocial: el papel de la cultura, la familia, la escuela y la personalidad. Revista Mexicana de Investigación en Psicología, 6(2), 146-157. Recuperado de: http://www. revistamexicanadeinvestigacionenpsicologia.com/

Garaigordobil, M., \& Berrueco, L. (2007). Efectos de un programa de intervención de 5 a 6 años: Evaluación del cambio proactivo en factores conductuales y cognitivos de desarrollo. SUMMA Psicológica, 4(2), 3-19.

González-Portal, M. (2000). Conducta prosocial: evaluación e intervención ( $3^{\mathrm{a}}$ Ed.) Madrid: Morata.

Gordillo, E. (2013). Agrupamiento escolar y frecuencia de conductas disruptivas en estudiantes de segundo grado de educación secundaria del Callao. Educación, 23(43), 91-112. Recuperado de: http://revistas. pucp.edu.pe/index.php/educacion

Gordillo, E., \& Gamero, G. (2013). Agrupamiento escolar y frecuencia de conductas disruptivas en estudiantes de segundo grado de Arequipa. Revista de Investigación, 4, 67-94.

Gustems, J., \& Calderón, C. (2014). Afrontamiento proactivo y bienestar psicológico en estudiantes de maestro en la Universidad de Barcelona. Programa de investigación de Docencia Universitaria (REDICE) de la 
Universitat de Barcelona (pp. 460-465). Recuperado de: http://eprints. ucm.es/

Herfst, S., van Oudenhoven, \& Timmerman, M. (2008). Intercultural effectiveness training in three Western immigrant countries: A cross-cultural evaluation of critical incidents. International Journal of Intercultural Relation, 32, 67-80. https://doi.org/10.1016/j. ijintrel.2007.10.001

Izon-Zintilini, M., \& Morelato-Giménez, G. (2008). Habilidades sociocognitivas en niños con conductas disruptivas y víctimas de maltrato. Universitas Psychologica 7(2), 357-367.

Mariñez, A. (2003). El sentido de la vida en la obra de Viktor Frankl. Madrid: Publidisa.

Matalinares, M., et al. (2013). Adicción al internet y agresividad en estudiantes de secundaria del Perú. Revista de Investigación en Psicología, 16(1), 75-93.

Mestré, M., Samper, P., \& Frías, M. (2002). Procesos cognitivos y emocionales predictores de la conducta prosocial y agresiva: La empatía como factor modulador. Psicothema, 14(2), 227-232.

Ministerio de Educación-Minedu (2014). Paz escolar. Estrategia nacional contra la violencia escolar ( $2^{\circ}$ Ed.). Ministerio de Educación: Lima.

Monereo, C. (2010). La formación del profesorado: una pauta para el análisis e intervención a través de incidentes críticos. Revista Iberoamericana de Educación 52, 149-178.

Monereo, C., \& Monte, M. (2011). Docentes en tránsito. Incidentes críticos en secundaria. Barcelona: Graó.

Muñoz, M., \& Nail, Ó. (2013). Incidentes críticos de aula: experiencia en escuelas de la Provincia de Concepción. En: Óscar Nail Kröyer (coord.). Análisis de incidentes críticos de aula. Una herramienta para el mejoramiento de la convivencia (pp. 51-74).RIL editores: Santiago de Chile.

Nagel, T. (2004). La posibilidad del altruismo ( $1^{\circ}$ Ed.). México D.F.: Fondo de Cultura Económica. 
Nail, Ó. (2013). Convivencia escolar en el aula. En: Óscar Nail Kröyer (coord.). Análisis de incidentes críticos de aula. Una herramienta para el mejoramiento de la convivencia. (pp. 15 - 36). Santiago de Chile: RIL editores.

Nail, Ó., Muñoz; M., \& Gajardo, J. (2013). Principios orientadores de la convivencia en el aula: una estrategia de reflexión colectiva. Educacăo e Pesquisa, 39(2), 376-385. http://dx.doi.org/10.1590/S151797022013000200006

Olweus, D. (2004). Conductas de acoso y amenazas entre escolares ( $2^{\mathrm{a}} \mathrm{Ed}$.) Madrid: Morata.

Olweus, D. (2005). Conductas de acoso y amenazas entre escolares (Tomo 14). México D.F.: Alfaomega.

Oficina Regional para América, Latina y el Caribe-UNICEF (2011). Violencia escolar en América Latina y el Caribe: Superficie y fondo. UNICEF: Panamá.

Puente, R. (2014). Relación entre conductas de riesgo e intimidación entre pares en estudiantes de secundaria (Tesis de maestría). Universidad Nacional Mayor de San Marcos, Lima.

Puente-Baella, R. (2008). Riesgo de conducta antisocial en adolescentes. En: Novella, A. y Roca, M. (Comp.). El crecimiento emocional del adolescente en la escuela. Lima: Centauro editores.

Publimetro.pe (agosto, 2015). Colegios registran más de 3 mil casos de violencia. Recuperado de: https://goo.gl/xQc2Am

Quintana, A., Montgomery, W., Malaver, C., \& Ruiz, G. (2011). Estilos explicativos y habilidades para la gestión de negociación de conflictos en adolescentes espectadores de violencia entre pares (bullying). Revista de Investigación del Instituto de Investigación en Psicología 14(1), 95108.

Real Academia Española-RAE (2014). Diccionario de la lengua española $\left(23^{\circ}\right.$ Ed.). Recuperado de: http://dle.rae.es/?id=LEEIbI5

Roche-Olivar, R. (2004). Inteligencia prosocial. Educación de las emociones y valores. Barcelona: Univertat Autónoma de Barcelona. 
Román, M., \& Murillo, F.J. (2011). América Latina: violencia entre estudiantes y desempeño escolar. Revista de la Comisión Económica para América Latina y El Caribe-Cepal, 104, 53-54. Recuperado de: http://www.cepal.org/es/publicaciones/tipo/revista-cepal

Sabariego, M., \& Bisquerra, R. (2004). Fundamentos metodológicos de la investigación educativa. En: Rafael Bisquerra Alzina (Coord.). Metodología de la investigación cientifica pp. 19-49. Córdoba: Brujas.

Sanz, N., de Miguel, J., \& Gómez, P. (2014). El efecto del deseo de control en el desarrollo de competencias. International Journal of Developmental and Educational Pyschology, 1(2), 345-352. http://dx.doi.org/10.17060/ ijodaep.2014.n1.v2.449

Schuster, B. (2009). Preventing, preparing for critical incidents in schools. NIJ Journal, 262, 44-46. Recuperado de: https://www.ncjrs.gov/ pdffiles1/nij/225765.pdf

Tripp, D. (2012). Critical incidents in teaching. Developing professional judgement. C. Ed. New York: Routledge-Taylor \& Francis Group.

Torres, L. Díaz, J., \& Pérez, E. (2012). Programación neurolingüística: herramienta comunicacional efectiva de un liderazgo proactivo. Revista electrónica de psicología Iztacala 15(3), 969-986 Recuperado de: http:// www.medigraphic.com/pdfs/epsicologia/epi-2012/epi123j.pdf

Vaello, J., \& Vaello, O. (2010). Proactividad interna: ejercicio de autoevaluación. Revista Padres y Maestros, 332, 30-33.

Valdés, A.M., \& Monereo, C. (2012). Desafíos a la formación del docente inclusivo: la identidad profesional y su relación con los incidentes críticos. Revista Latinoamericana de Educación Inclusiva, 6(2), 193208. Recuperado de: http://www.rinace.net/rlei/numeros/vol6-num2/ art8.pdf

Valdivieso, P. (2009). Violencia escolar y relaciones intergrupales, sus prácticas y significados en las escuelas secundarias públicas de la comuna de Peñalolén en Santiago de Chile (Tesis doctoral). Universidad de Granada, Granada.

Valongo, A., \& Suely, M. (2006). Incidentes críticos del proceso enseñanzaaprendizaje del curso de pregrado en enfermería según la percepción de 
alumnos y docentes. Revista Latinoamericana de Enfermagen, 14(5). http://dx.doi.org/10.1590/S0104-11692006000500016

Vallés, A. (2014). Emociones y sentimientos en el acoso escolar. Revista Digital EOS Perú, 3(1), 7-17.

Vallejos, R.-M. (2013). La técnica de análisis de incidentes críticos: origen y desarrollo. En: Óscar Nail Kröyer (coord.). Análisis de incidentes críticos de aula. Una herramienta para el mejoramiento de la convivencia (pp. 37-49) RIL editores: Santiago de Chile.

Villacorta, N. (2014). Situaciones de acoso escolar y su relación con el rendimiento académico en los estudiantes de secundaria de la I.E. Juan Espinoza Medrano en el año 2013 (Tesis de licenciatura). Universidad Nacional Mayor de San Marcos, Lima.

Xifra, J. (2009). Comunicación proactiva. La gestión de conflictos potenciales en las organizaciones. Barcelona: Gedisa. 